\title{
Measurements of the production of vector bosons in association with heavy flavour quarks at ATLAS.
}

\section{Georges AAD*}

On behalf of the ATLAS Collaboration

CPPM, Aix-Marseille Université and CNRS/IN2P3, Marseille, France

E-mail: aadecern.ch

\begin{abstract}
The production of heavy flavour quarks in association with a vector boson represents an important process to study quantum chromodynamics. High precision is achieved in measurements of differential cross sections and comparisons are made to state-of-the-art next-to-leading-order quantum chromodynamics calculations and different parton distribution function parameterizations. The measurement of the production of a $W$ boson in association with $b$ quarks or with a single charm quark using $4.6 \mathrm{fb}^{-1}$ of pp collisions at $\sqrt{s}=7 \mathrm{TeV}$ with the ATLAS detector is reported. The $W+c$ measurement is performed for events with a $W$ boson plus a tagged $c$-jet or a $D / D^{*}$ meson. $W+c$ cross sections integrated over a fiducial kinematic range with their impact on the $s$-quark density in the proton are reported.
\end{abstract}

XXII. International Workshop on Deep-Inelastic Scattering and Related Subjects 28 April - 2 May 2014

Warsaw, Poland

${ }^{*}$ Speaker. 


\section{Introduction}

The production of jets in association with a vector boson represents an important process to study quantum chromodynamics (QCD) and constrain parton distribution functions (PDFs) at hadron colliders. $W / Z$ production including heavy flavour quarks is known with a relatively limited precision from both the theoretical and the experimental sides. The production of a $W$ boson with either $b$ jets [1] or a single $c$ jet [2] and the production of a $Z$ boson with $b$ jets [3] are studied by the ATLAS experiment [4]. In this document, only measurements of the production of a $W$ boson with heavy flavour jets are included with focus on $W+c$ results. These measurements are performed with $4.6 \mathrm{fb}^{-1}$ of LHC data collected by the ATLAS detector at $\sqrt{s}=7 \mathrm{TeV}$ in 2011 .

\section{General analyses strategies}

The different $W+$ heavy-flavour analyses employ the same general strategy to extract the corresponding signal yields. $W$ bosons are identified with high- $p_{\mathrm{T}}$ isolated leptons (electrons or muons) and the presence of missing transverse energy corresponding to a neutrino from the $W$-boson decay. Various techniques are used to identify the presence of heavy flavour quarks. The signal yields extracted from data, after background subtraction, are corrected for trigger and reconstruction effects to obtain cross sections defined at particle level in a fiducial region chosen to reduce Monte Carlo (MC) based extrapolations.

\section{Production of a $W$ boson in association with bottom quarks}

In addition to the $W$ boson selection criteria listed in section 2, events are required to have exactly one or two jets. Exactly one jet is required to be identified as a $b$ jet with the CombNN (IP3D+JetFitter [5]) $b$-tagging algorithm. The veto on a second $b$-tagged jet is essential to reduce the large $t \bar{t}$ background.

Several processes contribute to the overall background for the $W+b$-jets signal. The $t \bar{t}$, single top and multijet backgrounds are estimated by means of data driven methods. Diboson and $Z+$ jets backgrounds are estimated from MC simulation. The $W+b$-jets, $W+c$-jets and $W+$ lightjets contributions are statistically separated by fitting the CombNN output distribution observed

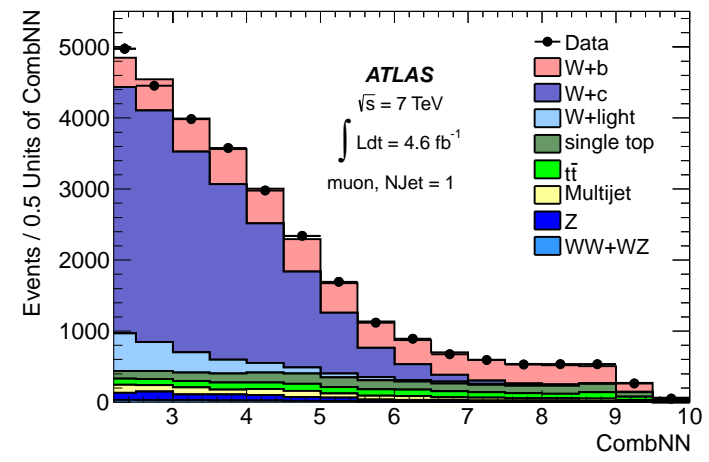

Figure 1: $\operatorname{CombNN}$ distributions for the $b$-tagged jet in data and MC simulation for the 1-jet analysis region in the muon channel. The MC samples are normalised to the results of the fit. in data. The contributions of $b, c$ and light jets produced with a $W$ boson are free parameters of the fit while the normalisation of other processes are allowed to float within their corresponding uncertainties. The CombNN distributions, normalised to the result of the fit, for events with exactly one jet in the muon channel are shown in figure 1 . 
The $W+b$-jets yields obtained from the CombNN fits are converted to fiducial cross sections for $W+b$-jets production times the branching ratio for each $W \rightarrow \ell v$ decay channel $(\ell=e, \mu)$ as explained in section 2. The results are compared to next-to-leading-order (NLO) predictions of MCFM and of POWHEG interfaced to PYTHIA , and to the predictions of ALPGEN , interfaced to HERWIG , that is scaled by the NNLO normalisation factor for the inclusive $W$ cross section. MCFM fixed order calculations are corrected for hadronisation and double-parton interaction effects. The various predictions are in good agreement with the measured cross sections within the experimental and the theoretical uncertainties.

Differential cross sections are also extracted, in the 1-jet and 2-jet regions, as a function of the transverse momentum of the leading $b$ jet. The measured differential cross section in the 1 -jet region is shown in figure 2. Data measurements exhibit larger cross sections compared to predictions at large $p_{\mathrm{T}}$. However the predictions are still compatible with data within experimental and theoretical uncertainties.

\section{Production of a $W$ boson in association with a single charm quark}

The production of a $W$ boson in association with a single charm quark in proton-proton collisions is described at leading order by the scattering of a gluon and a down-type quark $(d, s, b)$. At the LHC, $d$ and $b$ initiated processes are suppressed by the matrix elements $V_{c d}$ and $V_{c b}$. The $g s \rightarrow W c$ process and its higher-order corrections are dominant. Therefore the $p p \rightarrow W c X$ production is directly sensitive to the $s$-quark distribution function in the proton at momentum-transfer values on the order of the $W$-boson mass $\left(m_{W}\right)$.

The $s$-quark PDF has been determined by neutrino-nucleon deep inelastic scattering (DIS) experiments [6,7] at momentum transfer squared $Q^{2} \sim 10 \mathrm{GeV}^{2}$ and momentum fraction $x \sim 0.1$. Some analyses $[8,9,10]$ indicate that the $s$-quark sea is suppressed relative to the $d$-quark sea at all values of $x$ while others [11] suggest that $\mathrm{SU}(3)$ symmetry is restored as $x$ decreases. A recent analysis of inclusive $W$ and $Z$ production data from ATLAS and DIS data from HERA has bolstered the case for an SU(3)-symmetric sea at $x \sim 0.01$ [12]. The $W+c$ production cross section is measured at the Tevatron $[13,14,15]$ and by the CMS collaboration [16]. CMS exploited this measurement to constrain the $s$-quark PDF in ref. [17].

In events where a $W$ boson decays to an electron or muon, the charm quark is tagged either by the presence of a jet containing a soft muon stemming from the semileptonic decay of $c$ hadrons or by the presence of a $D$ or a $D^{*}$ hadron $\left(D^{(*)}\right)$. In $W+c$ production, the $W$ boson is always accompanied by a charm quark with charge of opposite sign: the charge of the soft muon or the 
$D^{(*)}$ meson is thus opposite to the $W$ boson charge and its corresponding decay lepton. Most backgrounds are evenly distributed between events with opposite-sign (OS) and same-sign (SS) charge. Therefore, the strategy used in this analysis is to determine the $W+c$ yields by measuring the difference between the number of opposite-sign and same-sign charge events (OS-SS).

\subsection{Identification of the charm quark using a charmed meson}

In events satisfying the $W$ selection criteria, $D^{(*)}$ mesons are reconstructed for the decay modes $D^{+} \rightarrow K^{-} \pi^{+} \pi^{+}$and $D^{*+} \rightarrow D^{0} \pi^{+}$ with $D^{0} \rightarrow K^{-} \pi^{+}, D^{0} \rightarrow K^{-} \pi^{+} \pi^{0}$, and $D^{0} \rightarrow$ $K^{-} \pi^{+} \pi^{-} \pi^{+}$and their charge conjugates. Combination with the correct charge assignment are considered. The distributions of the mass (for the $D$ ) or the mass difference $\Delta m=m\left(D^{*}\right)-m\left(D^{0}\right)$ (for the $D^{*}$ ) are formed in the OS and SS samples and subsequently computed for OS-SS events. The $W D^{(*)}$ yield is extracted for each decay mode by fitting the mass or mass difference distribution in OS-SS events. An example of the fit for $D^{*-}$ production including the signal and the background

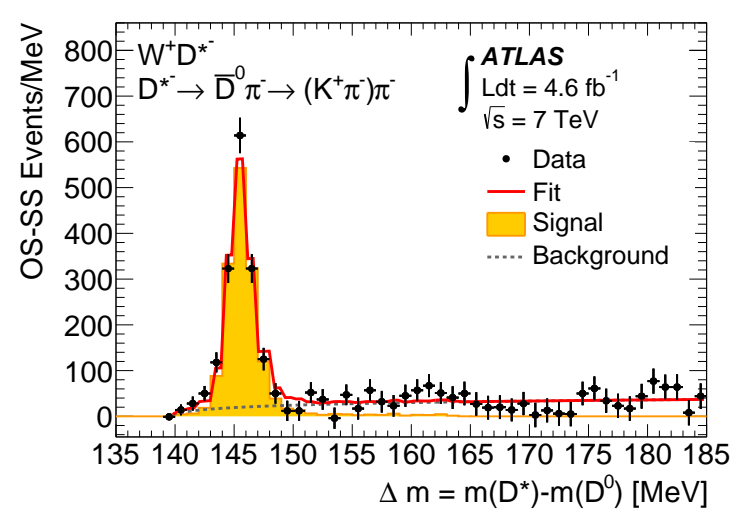

Figure 3: Fit example to the distributions of $\Delta m=m\left(D^{*}\right)-m\left(D^{0}\right)$ in OS-SS events. templates is shown in figure 3 .

\subsection{Identification of the charm quark using a soft muon}

Events passing the $W$ boson selection are required to contain one or two jets. Exactly one jet is required to be associated with a soft muon. The main backgrounds are the production of a $W$ or a $Z$ boson in association with light jets or multijet production. These backgrounds are estimated using data driven methods. Smaller backgrounds such as top and diboson production are estimated using Monte Carlo. $W$ boson production with a pair of $c$ or $b$ jets completely cancels for OS-SS events. The number of OS-SS signal events is computed by removing the estimated residual background yields from observed data events after the OS-SS subtraction.

\subsection{Results}

The $W D^{(*)}$ and $W c$-jet signal yields are used to determine fiducial cross sections times the branching ratio $W \rightarrow \ell v$ in a similar way to what is done for the $W+b$-jets analysis (section 3 ). The electron and muon channels and the different decay modes of the $D^{*}$ meson are averaged into single cross section measurements. The $W+c$-jets, $W+D$ and $W+D^{*}$ cross sections are kept as distinct measurements. The measured integrated fiducial cross sections is compared to theoretical predictions based on various PDF sets in figure 4. The theoretical predictions are obtained from aMC@NLO MC simulation that incorporates NLO QCD matrix-element calculations into a parton-shower framework. Herwig+ is used to model the parton shower, hadronisation and underlying event of the aMC@NLO simulation. The predictions obtained with the ATLASepWZ12 and NNPDF2.3COLL sets overlap with data; simulations using CT10, HERAPDF1.5 

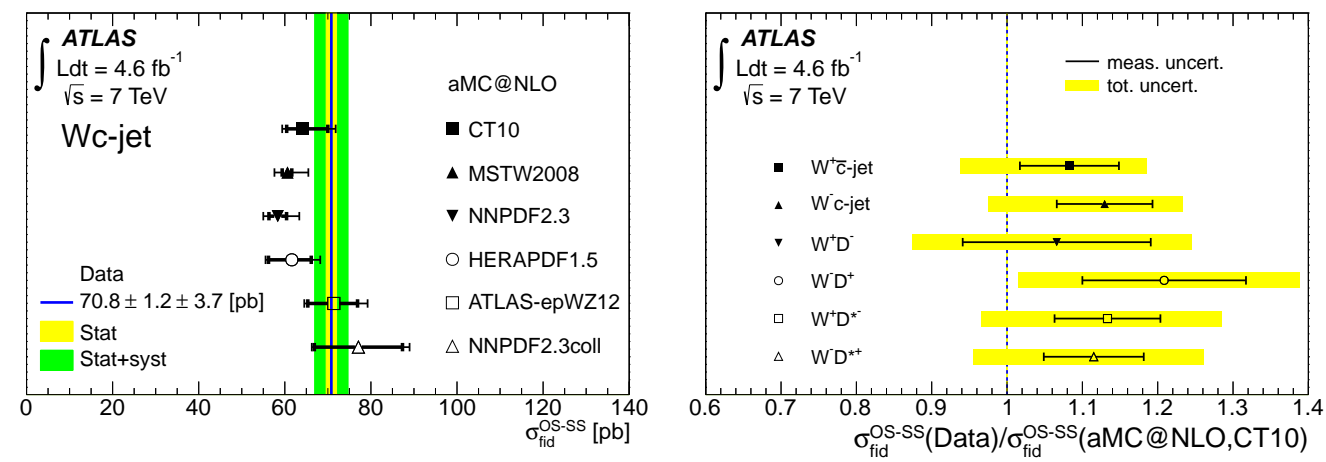

Figure 4: Left: measured fiducial cross section $\sigma_{\text {fid }}^{\mathrm{OS}-\mathrm{SS}}(W c$-jet $)$ compared to various PDF predictions based on aMC@NLO. The inner error bars on the theoretical predictions show the $68 \%$ confidence level uncertainties obtained from the error sets provided with each PDF set, while the outer error bar represents the total theoretical uncertainty. Right: data/theory cross section ratio for different cross section measurements. The predictions are taken from the aMC@NLO sample generated with the CT10 PDF set.

and MSTW2008 are also in agreement with the measurements. The prediction obtained with NNPDF2.3 is less favoured.

The compatibility between the measurements from different channels is illustrated in figure 4, which shows the ratios of the measured cross sections with respect to the corresponding theoretical predictions obtained from the CT10 PDF. The various measurements with different identification techniques of the presence of a $c$-quark are perfectly compatible within experimental uncertainties.

The measurements are performed separately for events with a positively and a negatively charged $W$ boson and the corresponding cross section ratios are computed. In addition differential cross sections are measured as function of the lepton pseudorapidity $\left(\left|\eta^{\ell}\right|\right)$. These measurements suffer from large statistical uncertainties and are compatible with the tested predictions.

\subsection{Constraint on parton density functions}

The $\chi^{2}$-minimisation procedure implemented in the HERAFitter framework [18, 19, $20,21]$ is used to assess the quantitative agreement between data and PDF predictions. The fit is applied to the measurements differential in $\left|\eta^{\ell}\right|$. Systematic and theoretical uncertainties are included as nuisance parameters in the fit. Correlations between measurements in different channels and bins are taken into account. All tested PDF sets are in agreement with data within the total uncertainties. However the fit favours PDFs with large $s$-quark density such as ATLAS-epWZ12 and NNPDF2.3COLL.

The HERAPDF1.5 PDF set models the $s$ -

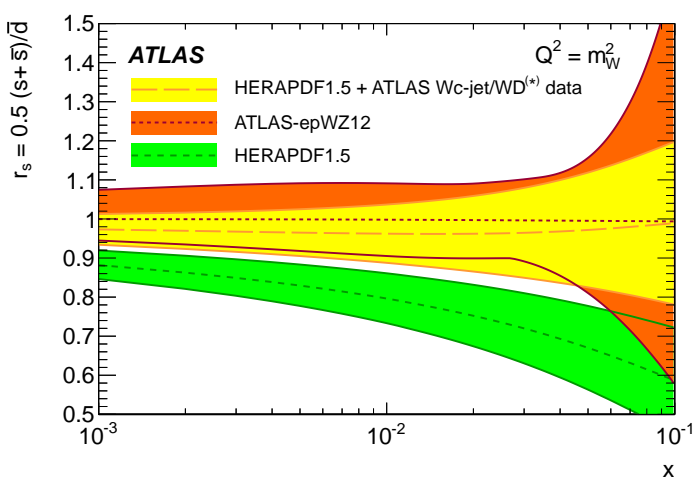

Figure 5: Ratio of strange-to-down sea-quark distributions $r_{s}=0.5(s+\bar{s}) / \bar{d}$ as a function of $x$. quark density by a single parameter describing the ratio between the $s$-quark sea and the $d$-quark 
sea densities in the proton. The $\chi^{2}$-minimisation procedure discussed above is used to calculate a value for the $s$-quark density by leaving the corresponding parameter free in the fit. The result is shown in figure 5 . The $W+c$ fit leads to an $s$-quark density similar to the $d$-quark sea density in agreement with the ATLAS-epWZ12 PDF set which includes ATLAS $W$ and $Z$ data.

\section{Conclusion}

The ATLAS experiment published a set of cross section measurements for the production of vector bosons with heavy flavour quarks. $W+b$-jets measurements are in agreement with NLO predictions. Three different techniques are used to identify the $c$-quark in $W+c$ events leading to compatible results. The $W+c$ measurements are consistent with a wide range of PDFs but favour PDFs with an SU(3)-symmetric light-quark sea.

\section{References}

[1] ATLAS Collaboration, JHEP 1306 (2013) 084 [arXiv: 1302 . 2929].

[2] ATLAS Collaboration, JHEP 1405 (2014) 068 [arXiv: 1402 . 6263].

[3] ATLAS Collaboration, arXiv:1404.7042 [hep-ex].

[4] ATLAS Collaboration, JINST 3 (2008) S08003.

[5] ATLAS Collaboration,. https://cds.cern.ch/record/1369219.

[6] NuTeV Collaboration, D. Mason et al., Phys. Rev. Lett. 99 (2007) 192001.

[7] NuTeV Collaboration, M. Goncharov et al., Phys. Rev. D 64 (2001) 112006 [arXiv: hep-ex/0102049].

[8] A. D. Martin, W. J. Stirling, R. S. Thorne, and G. Watt, Eur. Phys. J. C 63 (2009) 189-285 [arXiv:0901.0002].

[9] S. Alekhin, J. Blumlein, S. Klein, and S. Moch, Phys. Rev. D 81 (2010) 014032 [arXiv:0908.2766].

[10] NNPDF Collaboration, R. D. Ball et al., Nucl. Phys. B 809 (2009) 1-63 [arXiv: 0808 .1231].

[11] H.-L. Lai et al., Phys. Rev. D 82 (2010) 074024 [arXiv: 1007.2241 ].

[12] ATLAS Collaboration, Phys. Rev. Lett. 109 (2012) 012001 [arXiv:1203.4051].

[13] CDF Collaboration, T. Aaltonen et al., Phys. Rev. Lett. 100 (2008) 091803 [arXiv : 0711.2901 ].

[14] CDF Collaboration, T. Aaltonen et al., Phys. Rev. Lett. 110 (2013) 071801 [arXiv:1209.1921].

[15] D0 Collaboration, V. M. Abazov et al., Phys. Lett. B 666 (2008) 23-30 [arXiv: 0803 . 2259].

[16] CMS Collaboration, JHEP 1402 (2014) 013 [arXiv: 1310 .1138].

[17] CMS Collaboration, arXiv:1312.6283 [hep-ex].

[18] H1 and ZEUS Collaborations, F. D. Aaron et al., JHEP 01 (2010) 109 [arXiv: 0911.0884 ].

[19] HERAFitter, http://projects.hepforge.org/HeraFitter.

[20] H1 Collaboration, F. Aaron et al., Eur. Phys. J. C 64 (2009) 561-587 [arXiv : 0904 . 3513].

[21] F. James and M. Roos, Comput. Phys. Commun. 10 (1975) 343-367. 